\title{
Independent thickness and lateral size sorting of two- dimensional materials
}

\author{
Heyuan Zhou ${ }^{1}$, Junyang $\operatorname{Tan}^{1}$, Liusi Yang ${ }^{1}$, Jingyun Wang ${ }^{1}$, Baofu Ding ${ }^{1}$, Yikun Pan ${ }^{1}$, Xinghua Yu ${ }^{1}$, \\ Minsu Liu ${ }^{1}$, Chuang Yang ${ }^{1}$, Ling Qiu ${ }^{1}$, Hui-Ming Cheng ${ }^{1,2}$ and Bilu Liu ${ }^{1 *}$
}

\begin{abstract}
Two-dimensional (2D) materials possess unique thickness- and lateral-size-dependent properties. Many efforts have been devoted to obtaining $2 \mathrm{D}$ materials with narrow structure heterogeneity while it is still challenging to independently control their thickness and lateral size, limiting their widespread applications. Here, we develop a three-step method which achieves independent thickness and lateral size sorting of $2 \mathrm{D}$ materials. Taking $2 \mathrm{D} \mathrm{h}-\mathrm{BN}$ flakes as an example, their thickness and lateral size are independently sorted to different fractions with thicknesses smaller than $6 \mathrm{~nm}$. In addition, the 2D h-BN flakes possess narrow distributions of both thickness and lateral size. We further develop a force field extraction method and achieve scalable size sorting of $2 \mathrm{D}$ h-BN, which is universal for sorting other $2 \mathrm{D}$ materials including $\mathrm{MoS}_{2}$ and graphene oxide. This work reports an effective method to produce structure homogenous 2D materials and will help fundamental studies and applications of 2D materials where thickness and lateral size are of concern.
\end{abstract}

Keywords: two-dimensional materials, sorting, h-BN, $\mathrm{MoS}_{2}$, graphene oxide, thickness, lateral size

\section{INTRODUCTION}

Two-dimensional (2D) materials have received intense interest due to their unique layered structure and thickness- and lateral-size-dependent properties [1-4]. Physical properties such as band structure, usually vary greatly regarding to the thickness of $2 \mathrm{D}$ materials $[5,6] . \mathrm{MoS}_{2}$, for example, shows an indirect $(1.2 \mathrm{eV})$ to direct $(1.9 \mathrm{eV})$ band gap transformation with thickness decreasing from bulk to monolayer. Top-down exfoliation is powerful to produce $2 \mathrm{D}$ materials in a large quantity. Typically, the as-prepared 2D materials have broad distributions in lateral size $(L)$ and thickness $(T)$, which is not desirable for their applications. Besides lateral size and thickness, their aspect ratio, i.e., $L / T$, also plays a key role in the quality and applications of 2D material-assembled thin films [7-10], active site exposure of $2 \mathrm{D}$ material catalysts [11-13], functionalization of 2D material flakes [14-17] as well as the field-induced alignment order of 2D material liquid crystals $[18,19]$. For example, 2D materials with larger aspect ratios have more sensitive responses to external stimulation than smaller ones, which would facilitate applications in light modulation and display technology [20,21]. Achieving monodispersity in both lateral size and thickness of $2 \mathrm{D}$ materials is important to realize the full potential of their unique electronic, optical and catalytic properties [7].

Many efforts have been devoted to controlling the thickness and lateral size of $2 \mathrm{D}$ materials. For example, Hersam et al. [22-25] have reported that isopycnic density gradient ultracentrifugation (iDGU) method can precisely control the thickness (layer number) of $2 \mathrm{D}$ materials, while their lateral sizes are still broad. On the other hand, Coleman et al. reported the sedimentation rate based DGU (sDGU) [2,26] and liquid cascade centrifugation (LCC) [15,27-29] method for lateral size sorting of $2 \mathrm{D}$ materials. For the sDGU and LCC sorted 2D materials, their lateral size and thickness are usually correlated. In addition, the LCC method can realize size selection in terms of mean length $(\bar{L})$, while the distribution range (i.e., the standard deviation: $\sigma(\bar{L})$ ) of each fraction is relatively large $(\sigma(\bar{L}) / \bar{L}=0.46)[28]$. Note that current sorting methods can either control the thickness or lateral size of 2D materials rather than both. Therefore, precisely sorting $2 \mathrm{D}$ materials with a narrow distribution of both thickness and lateral size remains challenging.

\footnotetext{
${ }^{1}$ Shenzhen Geim Graphene Center, Tsinghua-Berkeley Shenzhen Institute \& Institute of Materials Research, Tsinghua Shenzhen International Graduate School, Tsinghua University, Shenzhen 518055, China

${ }^{2}$ Laboratory for Materials Sciences, Institute of Metal Research, Chinese Academy of Sciences, Shenyang 110016, China

* Corresponding author (email: bilu.liu@sz.tsinghua.edu.cn)
} 
Here, we report a method to finely sort both the thickness and the lateral size of 2D materials. The method consists of three steps including coarse size sorting, fine size sorting, and thickness sorting. The thickness and lateral size of the as-prepared 2D h-BN are independently sorted to different fractions with their thickness smaller than $6 \mathrm{~nm}$. And narrow distributions of both thickness $(\sigma(T) / T=0.22)$ as well as lateral size $(\sigma(L) / L=0.32)$ are achieved in the three-step sorted $2 \mathrm{D}$ h-BN. In addition, we develop a force field extraction (FFE) method to realize fine size sorting of $2 \mathrm{D}$ h-BN in large quantities, without the use of density gradient. We further verify the universality and efficiency of the FFE method in fine lateral size sorting of various $2 \mathrm{D}$ materials including $\mathrm{MoS}_{2}$ and graphene oxide (GO). This work provides an effective way to obtain homogenous $2 \mathrm{D}$ materials in terms of both thickness and lateral size, and may impact applications that require 2D materials with defined structures and properties.

\section{RESULTS AND DISCUSSION}

To achieve fine and independent thickness and lateral size control of exfoliated 2D materials, we develop a threestep sorting method. As shown in Fig. 1, the first step is to achieve coarse lateral size sorting by systematically changing the centrifugation speed and collecting the resulting sedimentations, which is based on the differences in sedimentation rates among various-sized flakes. After this step, each precipitated fraction of $2 \mathrm{D}$ materials has a relative narrow lateral size distribution. These precipitated fractions from the first step are subsequently redispersed in Pluronic F68 aqueous solution and subjected to the second step for fine size sorting. The Step 2 refines the lateral size distribution of $2 \mathrm{D}$ materials by retaining large flakes as sedimentations and achieves narrow distribution of lateral size. The long molecule chains of Pluronic F68 are efficient in providing steric hindrance and forming anhydrous shells to prevent $2 \mathrm{D} \mathrm{h}$-BN flakes from restacking in aqueous solution. The mechanism has been well-established with Pluronic F68 as surfactant in iDGU sorting $[22,24]$. Then, as the third step, the bottom part collected from the Step 2 is re-dispersed and goes through tens hours of iDGU sorting, and the distribution of the thickness of 2D materials is narrowed. We find that the sequence of the three-step sorting process is important, and changes of the sequence lead to a low throughput and ineffective sorting process. Overall, the developed three-step sorting method is capable of fine sorting of 2D materials in both thickness and lateral size.

We show the effectiveness of the developed three-step sorting method by using tip-sonication exfoliated 2D h$\mathrm{BN}$ as an example (see methods for details). The as-prepared 2D h-BN sample shows wide distributions in both thickness (from 0.6 to $20 \mathrm{~nm}$ ) and lateral size (from 20 to $1500 \mathrm{~nm}$ ) (Fig. 2a). Atomic force microscopy (AFM) images (Fig. 2b-d) of 2D h-BN samples after each sorting step evidently reflect the improvement in distributions of both thickness and lateral size. The as-prepared 2D h-BN sample with an average length of $324.7 \pm 233.4 \mathrm{~nm}$ (Fig. 2e) is firstly coarse size sorted through the Step 1 by collecting sedimentations in sequence after each round of centrifugation. For 6,000-8,000 rpm sedimentation from the Step 1, this fraction with an average length at $218.7 \pm$ $97.6 \mathrm{~nm}$ with $\sigma(L) / L=0.45$, confirms the effectiveness of size sorting of the LCC method (Fig. 2f). The collected sedimentation is re-dispersed and subjected to centrifugation twice with the same centrifugation time and speed as the Step 1. This step (FFE) aims at narrowing the distribution range of lateral size of $2 \mathrm{D}$ h-BN by collecting sedimentation again. After the Step 2, the lateral size of the 6,000-8,000 rpm sedimentation is further refined with a narrower size distribution range $(L=237.4 \pm 68.2 \mathrm{~nm})$ (Fig. 2g). Meanwhile, the distribution of the thickness of $2 \mathrm{D}$ h-BN also improves from $10.6 \pm 4.6 \mathrm{~nm}$ in the asprepared $2 \mathrm{D}$ h-BN to $5.8 \pm 2.1 \mathrm{~nm}$ after the Step 2 sorting (Fig. S1). Then iDGU is needed as the third step to realize
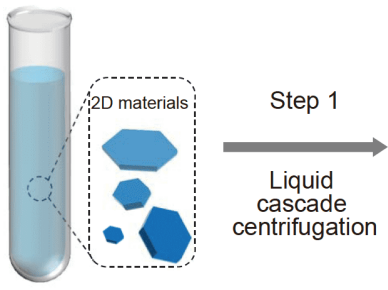

Coarse lateral size sorting
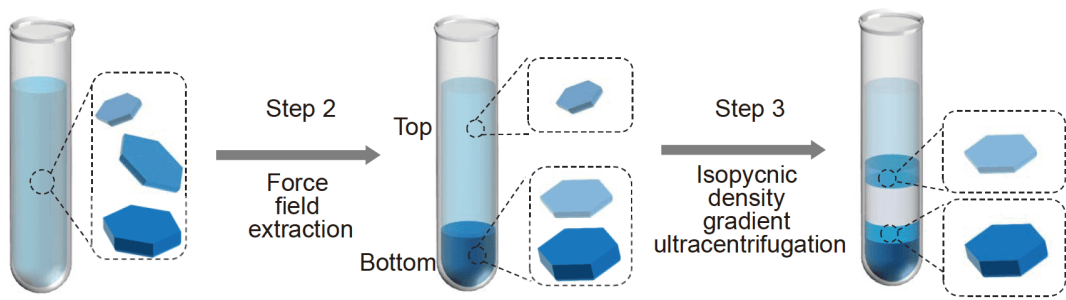

Fine lateral size sorting

Thickness sorting

Figure 1 Schematic of the three-step sorting method for fine and independent thickness and lateral size sorting of $2 \mathrm{D}$ materials. The method consists of coarse lateral size sorting by LCC (Step 1), fine lateral size sorting by FFE (Step 2), and thickness sorting by iDGU (Step 3). 

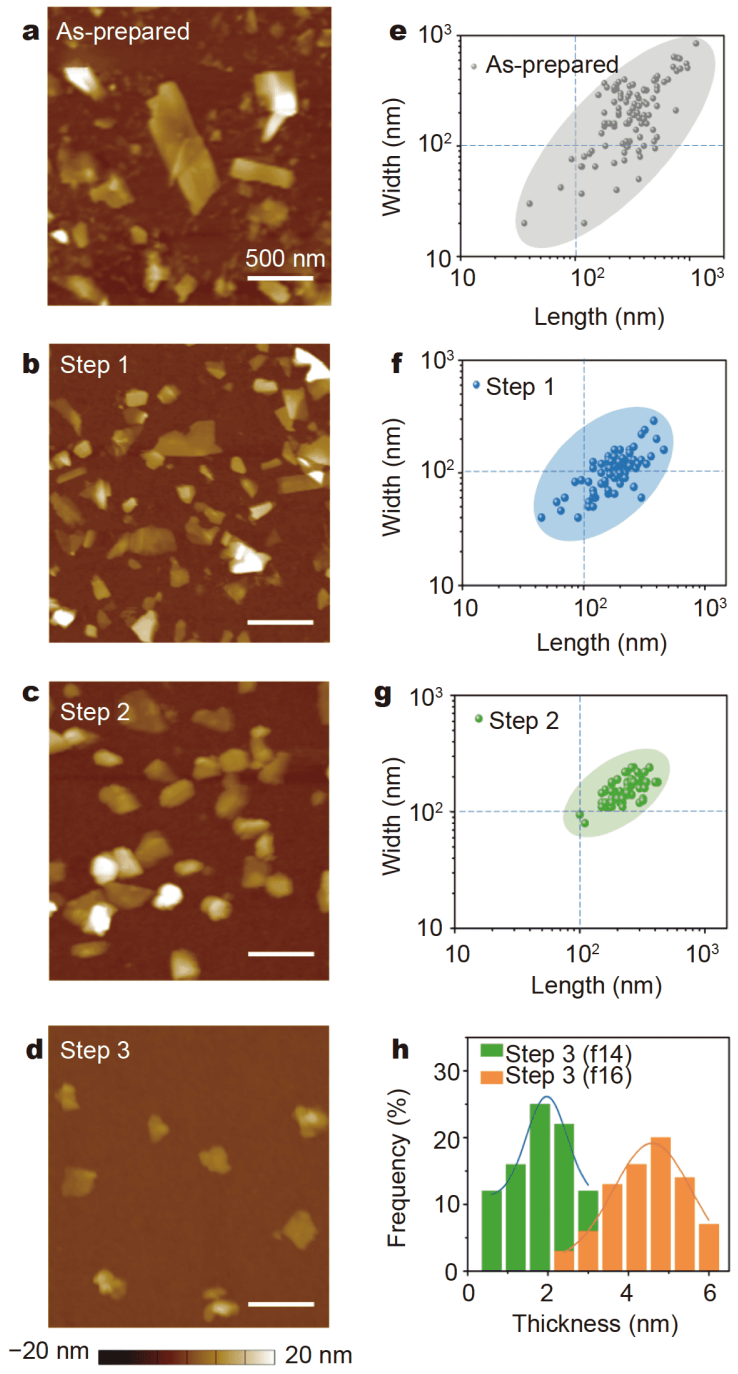

Figure 2 Fine and independent thickness and lateral size sorting of 2D h-BN. AFM images of (a) the original 2D h-BN and the samples after (b) Step 1, (c) Step 2, and (d) Step 3 sorting. Length and width distributions of (e) the original 2D h-BN, and the samples after (f) Step 1, and (g) Step 2 sorting, reflecting the narrowing of distribution in later size after Step 1 and Step 2 sorting. (h) Thickness distributions of 2D h-BN after Step 3 sorting, showing precise thickness control.

precise thickness sorting. With the $6,000-8,000 \mathrm{rpm}$ sedimentation from the Step 2 as an example, by re-dispersing in Pluronic F68 aqueous solution and going through tens hours of iDGU sorting with iodixanol as the density gradient, two clear white bands in snapshot represent the success of thickness sorting of $2 \mathrm{D} h-\mathrm{BN}$ (Fig. S2a). These bands are recovered in $1 \mathrm{~mm}$ steps using a piston gradient fractionator. These fractions are labeled as $f$, in which $n$ represents the depth position in the centrifuge tube. AFM statistical analyses show that these flakes are sorted into two separate fractions with the mean thickness value of $1.9 \pm 0.42 \mathrm{~nm}$ (f14) and $3.6 \pm$ $0.91 \mathrm{~nm}$ (f16) (Fig. 2h and Fig. S2b-c). To this end, with the completion of the three-step sorting, fine thickness and lateral size sorting of $2 \mathrm{D} \mathrm{h}-\mathrm{BN}$ is realized. The threestep method leverages the differences in sedimentation rates (Step 1 and Step 2) and buoyant densities (Step 3) to realize independent thickness and lateral size sorting of 2D materials. For the first time, the FFE sorting (Step 2) leverages the random distribution of $2 \mathrm{D}$ flakes induced by re-dispersion and the uneven relative centrifugation force in re-centrifugation for separation. The sequences of the three steps have also been carefully designed. Thus, the developed three-step method is effective in achieving narrow distributions in both thickness and lateral size of sorted 2D h-BN.

Next, we focus on the structure and quality of the sorted 2D h-BN flakes. Polymers, like Pluronic F68 and iodixanol, prevent us from obtaining precise thickness and lateral size values of the 2D materials. Therefore, we performed the polymer-removal by solvent exchange or thermal annealing before the characterization of $2 \mathrm{D}$ materials. After each step of sorting, the peaks in Raman spectra of h-BN remain almost identical at $1367 \mathrm{~cm}^{-1}$, which indicates the well-preserved structure and quality of h-BN (Fig. 3a). The optical band gaps of h-BN after each step of sorting remain at $5.8 \mathrm{eV}$, based on the plot of $(\alpha h v)^{2}$ versus photon energy $(h v)$, indicating the dispersed material is high-quality h-BN with its original structure (Fig. 3b). Fig. 3c shows the transmission electron microscope (TEM) image of h-BN flakes with the lateral size of $\sim 500 \mathrm{~nm}$ after the three-step sorting. The spacing of $0.22 \mathrm{~nm}$ between (100) planes is identified by high-resolution TEM (Fig. 3d), and the corresponding fast Fourier transform (FFT) image reveals the hexagonal lattice structure for h-BN. These results show that the pristine structure and quality of h-BN flakes are wellpreserved after the three-step sorting.

Then we focused on the necessity of the FFE sorting step in the three-step centrifugation. We systematically changed the centrifugation speed in Step 1 followed by the iDGU sorting without the FFE step. AFM images (Fig. S3) indicate that some unexpected small and thin flakes are collected as sedimentations even at low centrifugation speeds from Step 1 sorting (i.e., 2,000 and $4,000 \mathrm{rpm})$. We also witnessed small and thin flakes sediment at 1,000-2,000 rpm from Step 1 before iDGU step as shown in TEM images (Fig. S4). We refer this phenomenon as the "small flake wrapping effect". These unexpected small and thin flakes collected as sedimentations are much smaller than the average lateral size 

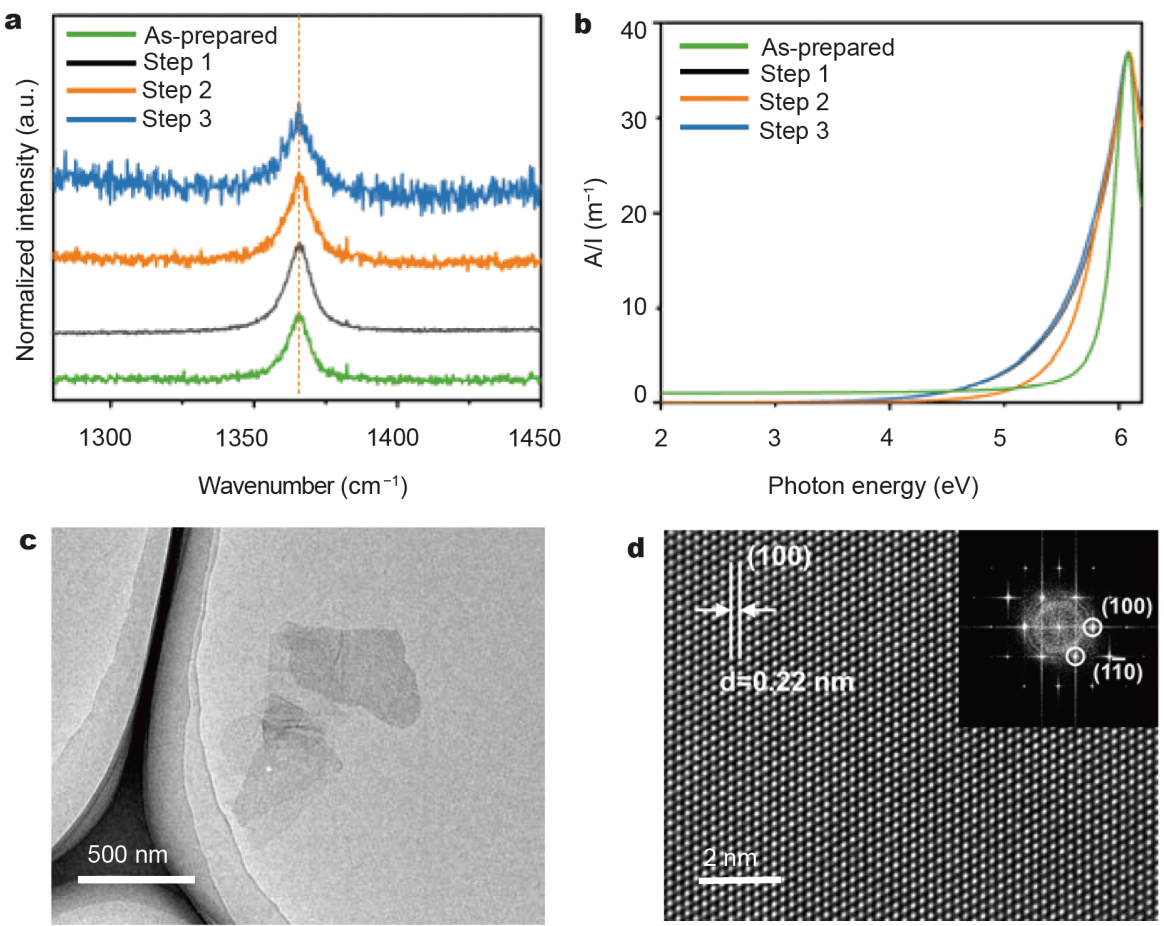

Figure 3 Characterization of the 2D h-BN after the three-step sorting. (a) Raman spectra of the as-prepared 2D h-BN and the samples after Step 1-3 sorting. (b) UV-Vis spectra of the as-prepared 2D h-BN and the samples after Step 1-3 sorting. (c) Low-magnification TEM image of the 2D h-BN after the three-step sorting. (d) High-resolution TEM image of the crystal quality and structure of the 2D h-BN after the three-step sorting. Inset: FFT image of $2 \mathrm{D}$ h-BN.

of that sedimentation, therefore causing the distribution of each size sorted fraction relatively large with $\sigma(L) / L=$ 0.46 . This is particularly dominating and noticeable if the LCC method directly combines with the iDGU sorting, since iDGU sorting generally screens out thick flakes. Thus, the introduction of the FFE step to eliminate the small flake wrapping effect is critical to achieving fine thickness and lateral size sorting of 2D materials (Fig. S5) in the developed three-step sorting method.

To elucidate the mechanism of the FFE sorting process, we studied the sedimentation rate of $2 \mathrm{D}$ materials based on the following equation:

$U=2\left(\rho_{\mathrm{p}}-\rho_{\mathrm{m}}\right)(r+t)^{2}\left(g^{\prime} / 9 \eta_{\mathrm{m}}\right)$.

According to previous reports [26,30,31], the sedimentation rate $(U)$ of colloidal particles with radius $(r)$ of core material in a given medium with density $\rho_{\mathrm{m}}$ and viscosity $\eta_{\mathrm{m}}$, in a centripetal force field of $g^{\prime}$, can be described by Equation (1). Here, $\rho_{\mathrm{p}}$ denotes the net density of the particle and $t$ denotes the thickness of the solvation shell. Since the LCC method is a density gradient-free sorting method, the $\rho_{\mathrm{m}}$ and $\eta_{\mathrm{m}}$ here are those of water (i.e., $\rho_{\mathrm{m}}=\rho_{\text {water }}, \eta_{\mathrm{m}}=\eta_{\text {water }}$ ), and $\rho_{\mathrm{p}}=\rho_{\mathrm{h}-\mathrm{BN}}=$ constant.
Therefore, the sedimentation rate $(U)$ of the particle dominantly depends on the $(r+t)^{2}$ term and $g^{\prime}$ term, which explains the differences in sedimentation rate among various-sized particles. Given the above analysis, the $g^{\prime}$ is further given by Equation (2) as relative centrifugation force (RCF) [32],

$g^{\prime}=\mathrm{RCF}=11.18 d\left(\frac{Q}{1000}\right)^{2}$,

where $Q=r p m$ representing the centrifugation speed and $d$ is the distance of the particle from the axis of rotation. The $g^{\prime}$ shows a linear relationship with $d$, reflecting the uneven $g^{\prime}$ along the centrifugation tube. In the bottom part of the centrifugation tube where $d$ is larger, the 2D materials will experience stronger RCF and therefore have larger sedimentation rate. Moreover, because of their initial position at the bottom part of the centrifugation tube, these $2 \mathrm{D}$ materials with large sedimentation rate only need to travel a short distance to precipitate as sedimentations (Fig. 4a). The FFE method consisted of redispersion of the collected sedimentation and re-centrifugation at the same centrifugation speed and time as the previous LCC step. We reasonably assume that the 

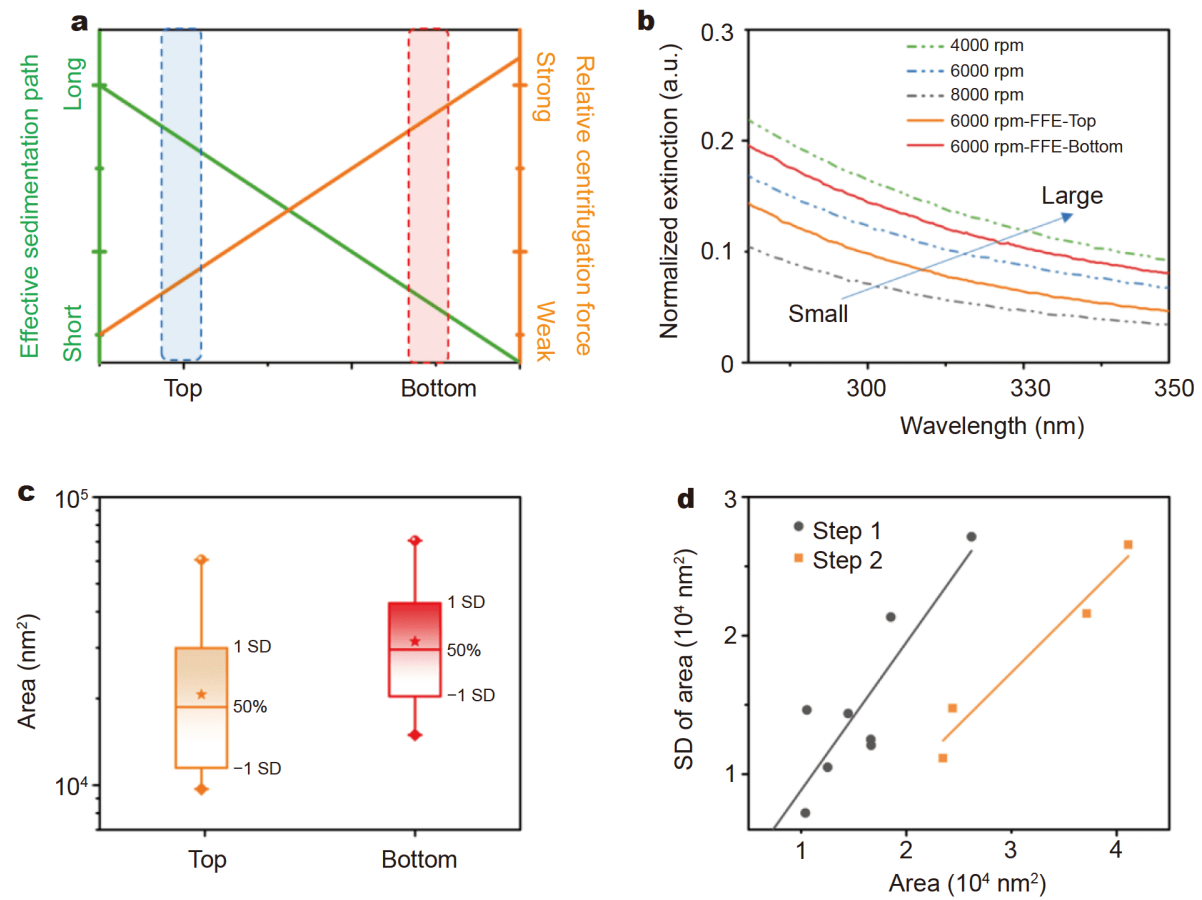

Figure 4 Size sorting of $2 \mathrm{D}$ h-BN by the FFE method. (a) Schematic of the effective sedimentation path and the relative centrifugation force in different positions (Top and Bottom part) of a centrifugation tube during the FFE process. (b) Normalized UV-Vis extinction spectra of the two fractions collected from the Top and Bottom part of centrifugation tube after FFE sorting at 6,000 rpm, indicating their different average lateral sizes. (c) Statistical maximum, minimum, mean, and standard deviation values of the area of $2 \mathrm{D}$ h-BN after the FFE sorting (the stars represent the mean values). (d) The standard deviation of area distribution versus the mean area of the sorted $2 \mathrm{D}$ h-BN.

collected sedimentation from the previous LCC step can randomly and homogeneously distribute throughout the centrifugation tube by full re-dispersion. Near the top part of the centrifugation tube, these wrapped small flakes would experience weak RCF and have a long effective sedimentation path. Hence, the wrapped small flakes are effectively extracted as supernatant fractions (top part) and a narrow distribution of the lateral size of $2 \mathrm{D}$ materials is achieved by collecting sedimentation (bottom part) again.

UV-Vis and AFM characterizations were used to support the proposed mechanism of FFE sorting. The shape of scattering background from UV-Vis spectra is highly dependent on the size of $2 \mathrm{D}$ h-BN. Ref. [27] and the comparison of UV-Vis curves of two fractions (Fig. 4b) from the FFE sorting confirm that the wrapped small flakes are effectively extracted in suspension fractions and the average lateral size of sedimentation becomes larger [27] due to the effective extraction of small wrapped flakes. The comparison of AFM statistical analyses of each fraction reaffirms that the relatively broad lateral size distribution has been divided into two fractions. Each fraction has a narrower size distribution with suspension fraction from the top part of centrifugation tube at $189 \mathrm{~nm}$ and sedimentation fraction from the bottom part at $314 \mathrm{~nm}$ (Fig. S6). The distribution range of the sorted 2D h-BN $(\sigma(\bar{L}) / \bar{L})$ decreases from 0.46 in Step 1 to 0.32 in Step 2. The change of areas $(\bar{A})$ of the $2 \mathrm{D}$ h-BN shares the same trend as that of $\bar{L}$. Fig. $4 \mathrm{c}$ shows that the maximum area of the $2 \mathrm{D}$ h-BN flakes in the bottom sedimentation fraction is around $60,000 \mathrm{~nm}^{2}$, while the small flakes with area $<20,000 \mathrm{~nm}^{2}$ are collected from the top suspension fraction, confirming the capability of the FFE sorting in extraction of the wrapped small flakes. The slope of the plotting (Fig. 4d) shows that the $\sigma(\bar{A}) / \bar{A}$ value has decreased from 0.75 (Step 1) to 0.45 (Step 2) which reconfirms a fine lateral size control of the FFE process compared with the LCC process. Taking together, leveraging the uneven RCF inside the centrifugation tube by re-dispersion and re-centrifugation, the FFE method enables the fine lateral size control by eliminating the small flake wrapping effect. The major difference between the FFE method and the existing sDGU and other methods is that FFE can strike a favorable balance be- 
tween sorting resolution and efficiency. In comparison, high sorting resolution of sDGU can be achieved, but at the expense of sorting efficiency with the majority volume of the centrifugation tube being taken up by the layout of step density gradient. On the other hand, LCC does not consider the initial random distribution of 2D flakes inside the centrifugation tube and the uneven relative centrifugation force field along the centrifugation tube.

We further explore the universality of the FFE sorting method in terms of the lateral size sorting of different 2D materials in a large quantity. Fig. $5 \mathrm{a}-\mathrm{c}$ show the $2 \mathrm{D}$ flakes prepared by different methods, including $2 \mathrm{D}$ h-BN prepared by the intermediate-assisted grinding exfoliation (iMAGE) [33], GO prepared by the electrochemical exfoliation and $\mathrm{MoS}_{2}$ prepared by lithium intercalation (see Supplymentary information for details). All these samples show a broad lateral size distribution right after exfoliation. After the FFE sorting, a narrow lateral size distribution is clearly witnessed for all three samples (Fig. 5d-f). The iMAGE prepared h-BN and electrochemically exfoliated GO are sorted to $L=1.2 \pm 0.3 \mu \mathrm{m}$ and $5.2 \pm 1.1 \mu \mathrm{m}$ with a narrow lateral size distribution of $\sigma(\bar{L}) / \bar{L}=0.28$ and 0.22 (Fig. $5 \mathrm{~g}, \mathrm{~h}$ ), respectively. The $\mathrm{MoS}_{2}$ is also sorted to $L=579.0 \pm 191.1 \mathrm{~nm}$ with a narrow lateral size distribution of $\sigma(\bar{L}) / \bar{L}=0.33$ (Fig. 5i). UV-Vis spectra and dynamic light scattering measurements (Fig. S7) reconfirm a better lateral size sorting resolution of $\mathrm{MoS}_{2}$ than the conventional LCC method. Compared with sDGU using density gradients $[2,26]$ to achieve the similar narrow lateral size distribution $(\sigma(\bar{L}) / \bar{L} \sim 0.3)$, the FFE method does not need to use density gradients, thus greatly simplifying the process. Due to the density gradient-free feature of the FFE method, the volume limitation of the samples in sDGU sorting (normally $10 \%$ of the gradient volume) no longer exists, meaning a faster sorting rate of the FFE process than the sDGU, which is necessary for scalable sorting of 2D materials (Fig. 5j-1).

\section{CONCLUSIONS}

We developed a three-step sorting method to achieve independent thickness and lateral size sorting of $2 \mathrm{D}$ materials. By using this method, the thickness and lateral size of the exfoliated h-BN are independently sorted into different fractions with $2 \mathrm{D}$ h-BN flakes thinner than $6 \mathrm{~nm}$. In addition, narrow distributions of both thickness $(\sigma(T) / T=0.22)$ and lateral size $(\sigma(L) / L=0.32)$ are
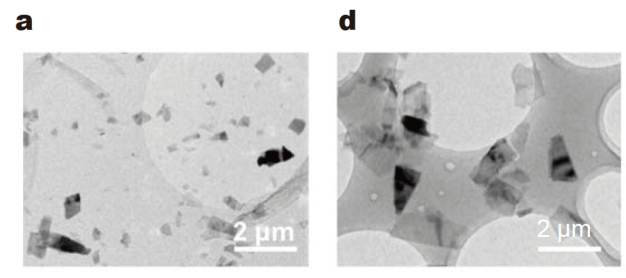

b

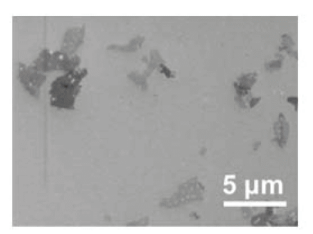

c

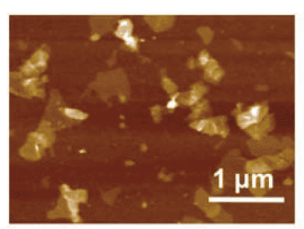

e

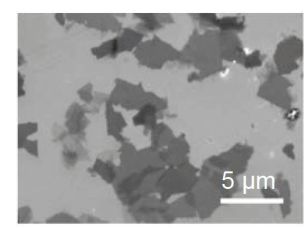

f

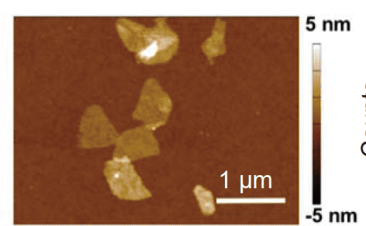

g

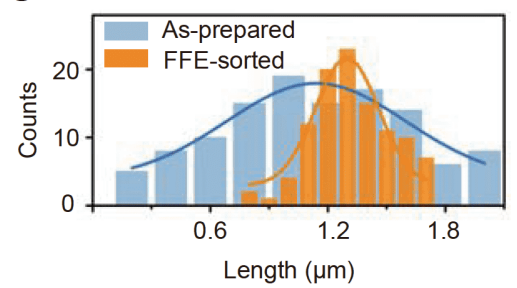

h

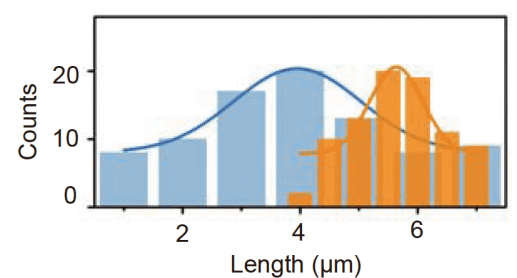

i

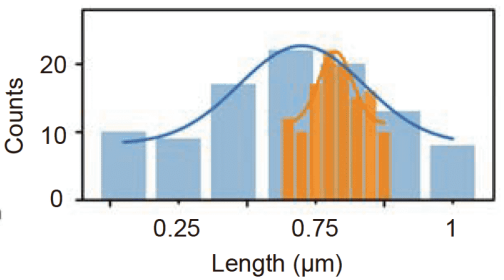

j

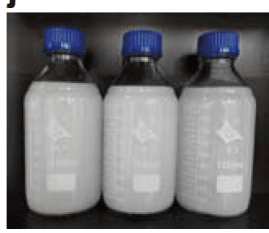

k

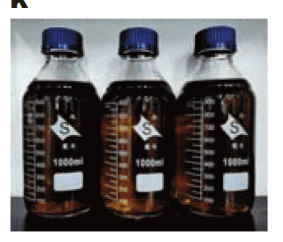

I

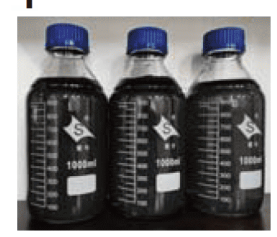

Figure 5 Universality and scalability of the FFE method for lateral size sorting of 2D materials. Typical images of the original and the FFE-sorted (a, d) h-BN, (b, e) GO and (c, f) $\mathrm{MoS}_{2}$. Statistical lateral size distributions of the as-prepared and FFE-sorted (g) h-BN, (h) GO and (i) MoS 2 . Digital photos of the FFE-sorted (j) h-BN, (k) GO and (l) $\mathrm{MoS}_{2}$ dispersions. Each container has $1 \mathrm{~L}$ dispersions of 2D materials. 
realized simultaneously. We also show the universality and efficiency of the FFE method in lateral size sorting of various $2 \mathrm{D}$ materials including $\mathrm{h}-\mathrm{BN}, \mathrm{GO}$ and $\mathrm{MoS}_{2}$ in large volumes. This work reports a method for obtaining thickness and lateral size homogenous 2D materials, which will stimulate their wide-range applications where narrow structure and property heterogeneity are required.

\section{Received 19 February 2021; accepted 21 April 2021;} published online 7 June 2021

1 Geim AK, Novoselov KS. The rise of graphene. Nat Mater, 2007, 6: 183-191

2 Backes C, Smith RJ, McEvoy N, et al. Edge and confinement effects allow in situ measurement of size and thickness of liquid-exfoliated nanosheets. Nat Commun, 2014, 5: 1

3 Cai Z, Liu B, Zou X, et al. Chemical vapor deposition growth and applications of two-dimensional materials and their heterostructures. Chem Rev, 2018, 118: 6091-6133

4 Ren J, Teng C, Cai Z, et al. Controlled one step thinning and doping of two-dimensional transition metal dichalcogenides. Sci China Mater, 2019, 62: 1837-1845

5 Li H, Zhang Q, Yap CCR, et al. From bulk to monolayer $\mathrm{MoS}_{2}$ : Evolution of Raman scattering. Adv Funct Mater, 2012, 22: 13851390

6 Xiao Y, Zhou M, Liu J, et al. Phase engineering of two-dimensional transition metal dichalcogenides. Sci China Mater, 2019, 62: 759775

7 Kang J, Sangwan VK, Wood JD, et al. Solution-based processing of monodisperse two-dimensional nanomaterials. Acc Chem Res, 2017, 50: 943-951

8 Ling Z, Wang Z, Zhang M, et al. Sustainable synthesis and assembly of biomass-derived $\mathrm{B} / \mathrm{N}$ co-doped carbon nanosheets with ultrahigh aspect ratio for high-performance supercapacitors. Adv Funct Mater, 2016, 26: 111-119

$9 \mathrm{He} \mathrm{Q}$, Zeng Z, Yin Z, et al. Fabrication of flexible $\mathrm{MoS}_{2}$ thin-film transistor arrays for practical gas-sensing applications. Small, 2012, 8: 2994-2999

10 Zhao Y, Xie Y, Liu Z, et al. Two-dimensional material membranes: An emerging platform for controllable mass transport applications. Small, 2014, 10: 4521-4542

11 Luo Y, Tang L, Khan U, et al. Morphology and surface chemistry engineering toward $\mathrm{pH}$-universal catalysts for hydrogen evolution at high current density. Nat Commun, 2019, 10: 269

12 Seh ZW, Kibsgaard J, Dickens CF, et al. Combining theory and experiment in electrocatalysis: Insights into materials design. Science, 2017, 355: eaad4998

13 Ren $\mathrm{S}, \mathrm{Yu} \mathrm{Q}, \mathrm{Yu} \mathrm{X}$, et al. Graphene-supported metal single-atom catalysts: A concise review. Sci China Mater, 2020, 63: 903-920

14 Sun X, Liu Z, Welsher K, et al. Nano-graphene oxide for cellular imaging and drug delivery. Nano Res, 2008, 1: 203-212

15 O'Neill A, Khan U, Coleman JN. Preparation of high concentration dispersions of exfoliated $\mathrm{MoS}_{2}$ with increased flake size. Chem Mater, 2012, 24: 2414-2421

16 Chaurasia A, Verma A, Parashar A, et al. Experimental and computational studies to analyze the effect of h-BN nanosheets on mechanical behavior of h-BN/polyethylene nanocomposites. J Phys Chem C, 2019, 123: 20059-20070
17 Peng L, Zhu Y, Li $\mathrm{H}$, et al. Chemically integrated inorganicgraphene two-dimensional hybrid materials for flexible energy storage devices. Small, 2016, 12: 6183-6199

18 Jalili R, Aminorroaya-Yamini S, Benedetti TM, et al. Processable 2D materials beyond graphene: $\mathrm{MoS}_{2}$ liquid crystals and fibres. Nanoscale, 2016, 8: 16862-16867

19 Ostapenko T, Nastishin YA, Collings PJ, et al. Aggregation, pretransitional behavior, and optical properties in the isotropic phase of lyotropic chromonic liquid crystals studied in high magnetic fields. Soft Matter, 2013, 9: 9487-9498

20 Aboutalebi SH, Gudarzi MM, Zheng QB, et al. Spontaneous formation of liquid crystals in ultralarge graphene oxide dispersions. Adv Funct Mater, 2011, 21: 2978-2988

21 Ding B, Kuang W, Pan Y, et al. Giant magneto-birefringence effect and tuneable colouration of 2D crystal suspensions. Nat Commun, 2020, 11: 3725

22 Kang J, Seo JWT, Alducin D, et al. Thickness sorting of twodimensional transition metal dichalcogenides via copolymerassisted density gradient ultracentrifugation. Nat Commun, 2014, 5: 5478

23 Kang J, Sangwan VK, Wood JD, et al. Layer-by-layer sorting of rhenium disulfide via high-density isopycnic density gradient ultracentrifugation. Nano Lett, 2016, 16: 7216-7223

24 Zhu J, Kang J, Kang J, et al. Solution-processed dielectrics based on thickness-sorted two-dimensional hexagonal boron nitride nanosheets. Nano Lett, 2015, 15: 7029-7036

25 Green AA, Hersam MC. Solution phase production of graphene with controlled thickness via density differentiation. Nano Lett, 2009, 9: 4031-4036

26 Sun X, Luo D, Liu J, et al. Monodisperse chemically modified graphene obtained by density gradient ultracentrifugal rate separation. ACS Nano, 2010, 4: 3381-3389

27 Griffin A, Harvey A, Cunningham B, et al. Spectroscopic size and thickness metrics for liquid-exfoliated h-BN. Chem Mater, 2018, 30: 1998-2005

28 Backes C, Szydłowska BM, Harvey A, et al. Production of highly monolayer enriched dispersions of liquid-exfoliated nanosheets by liquid cascade centrifugation. ACS Nano, 2016, 10: 1589-1601

29 Han JT, Jang JI, Kim SH, et al. Size sorting of chemically modified graphene nanoplatelets. Carbon Lett, 2013, 14: 89-93

30 Sun X, Tabakman S, Seo WS, et al. Separation of nanoparticles in a density gradient: FeCo@C and gold nanocrystals. Angew Chem, 2010, 121: 957-960

31 Price CA. Centrifugation in Density Gradients. Cambridge: Academic Press, 1982: 30-31

32 Graham JM. Biological Centrifugation. Oxford: Bios Scientific Publisher, 2001: 2-4

33 Zhang C, Tan J, Pan Y, et al. Mass production of 2D materials by intermediate-assisted grinding exfoliation. Natl Sci Rev, 2020, 7: 324-332

Acknowledgements This work was supported by the National Natural Science Foundation of China (51920105002, 51991340, and 51991343), Guangdong Innovative and Entrepreneurial Research Team Program (2017ZT07C341), the Bureau of Industry and Information Technology of Shenzhen for the "2017 Graphene Manufacturing Innovation Center Project" (201901171523), Shenzhen Basic Research Project (JCYJ20190809180605522, JCYJ20200109144620815 and JCYJ20200109144616617), and China Postdoctoral Science Foundation (2020M680540). 
Author contributions Zhou H and Liu B conceived the idea. Zhou H performed the $2 \mathrm{D}$ material sorting experiments and performed the Raman, UV-Vis and AFM characterizations. Tan J performed the TEM characterization. Liu M, Yang $\mathrm{C}$ and Yang $\mathrm{L}$ performed all the $2 \mathrm{D}$ material exfoliations. Ding B, Pan Y, Yu X, Wang J and Qiu L took part in the characterization data discussion. Liu B and Cheng HM supervised the project and directed the research. Zhou H and Liu B interpreted the results and wrote the manuscript with feedbacks from the other authors.

Conflict of interest Patents related to this research have been filed by Tsinghua-Berkeley Shenzhen Institute, Tsinghua University. The University's policy is to share financial rewards from the exploitation of patents with the inventors.

Supplementary information Experimental details and supporting data are available in the online version of the paper.

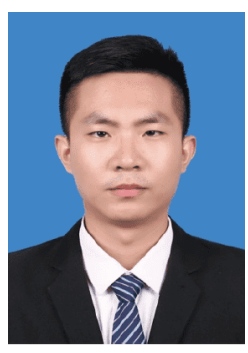

Heyuan Zhou is currently a Master student in the Low Dimensional Materials and Device Lab under the supervision of Prof. Bilu Liu at Tsinghua-Berkeley Shenzhen Institute, Tsinghua University. His research topic is about the sorting of solution-processed two-dimensional materials.

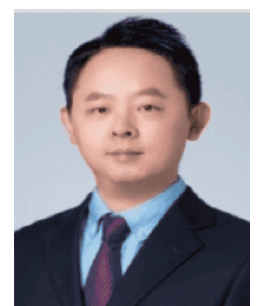

Bilu Liu obtained BSc degree from the University of Science and Technology of China (USTC, 2006), and $\mathrm{PhD}$ from the Institute of Metal Research, Chinese Academy of Sciences (2012). He worked at the University of Southern California as a Postdoctoral Researcher and later Research Assistant Professor (2012-2016). He joined Tsinghua-Berkeley Shenzhen Institute, Tsinghua University, in 2016 and now a tenured Associate Professor at Tsinghua Shenzhen International Graduate School, Tsinghua University. His research interests cover chemistry and materials science of low-dimensional materials with emphasis on the preparation and applications of carbon and 2D materials.

\section{二维材料厚度与片径的独立篮分}

周赫元 ${ }^{1}$, 谭隽阳 ${ }^{1}$, 杨柳思 ${ }^{1}$, 王婧云 ${ }^{1}$, 丁宝福 ${ }^{1}$, 潘意坤 ${ }^{1}$,

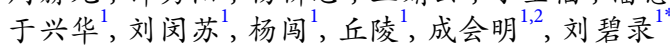

摘要 二维材料是当前科学研究的前沿领域. 二维材料的性质与其 厚度和片径密切相关, 如何获得厚度和片径均匀且可调控的二维 材料, 是其应用的关键. 本文提出了一种三步篮分法, 实现了二维 材料厚度与片径的独立笁分. 以二维六方氮化硼为例, 三步笁分法 可以将其厚度和片径独立地篮分为不同组分, 所得组分均具有较 窄的厚度和片径分布, 且厚度全部小于 $6 \mathrm{~nm}$. 本文还进一步发展了 一种力场提取法, 实现了二维六方氮化硼、二硫化钼和氧化石墨 烯的规模化片径篎分, 是二维材料片径篎分的普适方法. 本工作提 出的笁分方法可制备出结构均一的二维材料, 对其基础研究与应 用意义深远. 